\title{
Adaptive Thresholding Methods for Documents Image Binarization
}

\author{
Bilal Bataineh $^{1}$, Siti N.H.S. Abdullah ${ }^{2}$, K. Omar ${ }^{3}$, and M. Faidzul ${ }^{3}$ \\ Center for Artificial Intelligence Technology \\ Faculty of Information Science and Technology \\ Universiti Kebangsaan Malaysia, 43600, Bangi, Selangor, Malaysia \\ ${ }^{1}$ bilal_bataineh82@yahoo.com, $\left\{{ }^{2} \mathrm{mimi},{ }^{3} \mathrm{ko}, \mathrm{mfn}\right\}$ @ft.sm.ukm.my
}

\begin{abstract}
Binarization process is easy when applying simple thresholding method onto good quality image. However, this task becomes difficult when it deals with degraded image. Most current binarization methods involve complex algorithm and less ability to recover important information from a degradation image. We introduce an adaptive binarization method to overcome the state of the art. This method also aims to solve the problem of the low contrast images and thin pen stroke problems. It can also enhance the effectiveness of solving all other problems. As well as, it does not need to specify the values of the factors manually. We compare the proposed method with known thresholding methods, which are Niblack, Sauvola, and NICK methods. The results show that the proposed method gave higher performance than previous methods.
\end{abstract}

Keywords: binarization, document image, thresholding method, local binarization.

\section{Introduction}

This binarization of document images is a necessary step in pre-processing stage of document analysis applications. However, Kefali et al. claimed that the aim of binarization is to reduce unwanted information to increase the visibility of the desired information [1]. The process of binarization divides the values of pixels in the image into two levels such as black pixels represent as the foreground whereas the white pixels represent as the background. Based on previous studies [1-2], the binarization techniques are classified into two ways. Firstly, it involves hybrid or complex algorithms based on compound steps and existing techniques [3] and secondly, it applies simple or automatic thresholding methods to determine the thresholding value [4]. Comparing to both forms, the simple methods are easier to design and implement, also they give higher performance in different cases. In general, the simple thresholding methods are classified into two categories [1-2]: local thresholding methods [4] and global thresholding methods [5]. The local thresholding methods determine different thresholding values based on region of interest of an image. On the other hand, the global methods determine a single thresholding value for the whole image.

Kefali et al. has conducted an assessment about twelve outstanding methods on historical Arabic document images [1]. Similar to above competition, they used 150 
images containing different problems. The result showed that Nick and Sauvola methods achieved the first and second place respectively. Both the Nick and Sauvola fall into simple local thresholding method. They claimed that Nick method could perform extremely good, because it is based on thresholding shifting value whilst Sauvola's method worked better on solving the binarization noise. This initial study is one of the rare studies that focused on the historical Arabic documents.

Apart from that, Stathis et al. have written a deep evaluation paper based on a binarization competition [2]. At the same time, they also proposed a new evaluation technique namely pixel error. The competition involved about 30 well-known algorithms from different categories such as global, local and hybrid. Each method was tested on 150 different level of degraded document images. The competition results indicated that Sauvola method was outperformed on maximum intensity document images, whereas Johansen method obtained the best accuracy on normal intensity document images. As a result, this competition has given a clear view on the binarization methods categories and performance. Furthermore, we can also conclude that most prominent recent methods are only sufficient to tackle specific image cases.

As conclusion, we can assume that simple method outperform compared to hybrid or complex method. Unlike others, the simple method does not require high complexity cost. Nevertheless, it is an independence method that does not require other process in advance. Also, it is easy to construct and implement. On the other view, the simple thresholding methods can extraordinarily outperform if they the image includes preprocessing. Otherwise, the simple global thresholding approach can become ineffective on degraded images such as poor quality, illumination inconsistency and scanning error $[1,3]$.

Generally, performance of binarization process affects document image properties. Ntogas have summed up those challenges as: dirty spots, poor quality, low contrast between text and background (Fig.1 (a)), multi-color, thin strokes of pen, ink seeping from other documents and multi-size text [6] (Fig.1 (b, c)). In general, each method can deal well with some of these challenges but fails with the other.

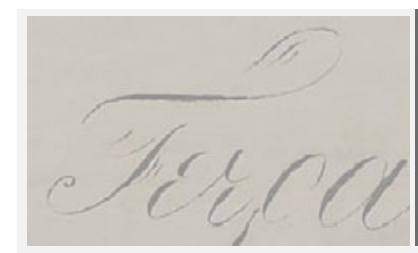

(a)

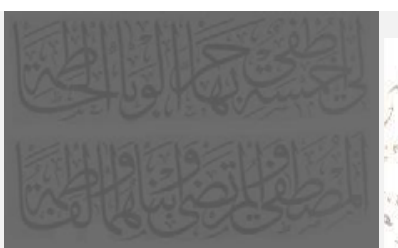

(b)

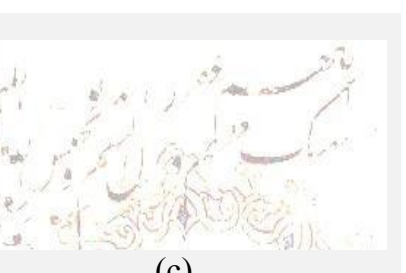

(c)

Fig. 1. (a) is a thin pen stroke and (b, c) a low contrast image

The aim of this work is to propose a binarization method for document images. This method adopts local approach to find the thresholding value of the windows in the document images. It has been achieved by introducing an adaptive thresholding method that able to find automatic thresholding value based on each window. The proposed method aims to solve the low contrast images and thin pen stroke problems. We compare the proposed method with other current existing methods such as Niblack, Sauvola, and NICK methods. We test them using selected images for visual experiments and other 
benchmark dataset with evaluation techniques for binarization methods. This paper is organized as follows. Section 2 reviews on the state of art of the most local binarization methods used. Section 3 explains the proposed method, Section 4 presents and analyses the experimental results. Finally, conclusions are presented in Section 5.

\section{The State of Art}

In this study we emphasize on simple and local thresholding approach. Out of many available techniques, we select only the most outperform and recent methods. They are Niblack, Sauvola and the NICK methods.

\subsection{Niblack's Method}

This method is proposed by Niblack in 1986 [7]. The thresholding value for each window is determined based on mean, $m$ and standard deviation, $\sigma$ values of pixels in that window as the following:-

$$
T=m+k \times \sigma,
$$

where $k$ is -0.2 by suggested by Niblack [7], and the window size are pre-determined by the user. Based on experiments, this method can strongly identify the text body. However, it also generate binarization black noise in empty windows.

\subsection{Sauvola's Method}

This method has been proposed by Sauvola et al. in 1997 [8]. This approach is actually inherited from Niblack method. It can successfully overcome the black noise problem. The thresholding formula is as following:-

$$
T=m \times\left(1-k \times\left(1-\frac{\sigma}{R}\right),\right.
$$

where $\mathrm{k}$ is a control factor in the range of $[0.2,0.5], \mathrm{R}$ is a predetermined image graylevel value. The author suggested $\mathrm{k}=0.2, \mathrm{R}=125$. Unfortunately, this method is less effective when image contrast between the text and background is relatively small.

\subsection{Nick Method}

This method is proposed by Khurshid et al. in 2010 [5]. NICK method was developed from Niblack method. It tried to solve low contrast problem by shifting down the thresholding value. The thresholding formula is as the following:-

$$
T=m+k \times \sqrt{\frac{\left(\sum P_{i}^{2}-m^{2}\right)}{N P}},
$$

where $k$ is a control factor in the range of $[-0.1,-0.2], P_{i}=$ the image pixel grey-scale value and $N P=$ the total pixels number in the image. The author suggested the $\mathrm{k}=-0.1$ [7]. Kefali et al. [1] claimed that NICK method gave the best performance compared with previous methods. However, low contrast images problem are still remained unsolved. 
In general, neither of these methods able to deal with all the problems. The methods deal well on some problems and fail on others. Furthermore, most of recent methods requires manual parameters/factors setting. Prior to this limitation, we introduce an automatic parameter setting for determining adaptive threshold value.

\section{The Proposed Method}

We aim to solve the problems and weaknesses of the previous. To achieve that, two factors have used in our method. The first one is the mean value, $m_{g}$ of all image's pixels, the other one is the adaptive standard deviation $\sigma_{\text {Adaptive }}$.

In Niblack method, the different properties of each windows led to binarization problem, whereas the binarization noises found in empty window. To solve this problem, the global mean value is used to round the extremist values of windows. In Sauvola and NICK methods, binarization faced problems when the contrast of the image is low. In images representation, the contrast value of images is denoted by the standard deviation $\sigma$. If image contrast is low, the standard deviation value will be too small and does not effective in the binarization process. To solve this problem, we adapted the standard deviation values of each image. Which leads to an equal effect regardless of the images contrast value. To present the proposed thresholding method on all gray-scale values, we fixed pixels values in the range of $[0,1]$. Where, the minimum scale value are 0 and the maximum scale value are 1 . Then, we define the proposed method formula as follows:

$$
T=m_{W}-\frac{m_{W}^{2}-\sigma_{W}}{\left(m_{g}+\sigma_{W}\right) \times\left(\sigma_{\text {Adaptive }}+\sigma_{W}\right)},
$$

where, $T$ is the thresholding value, $m_{W}$ is the mean value of the widow's pixels, $\sigma_{W}$ is the standard deviation of the widow's pixels, $m_{g}$ is the mean value of all pixels in the image and $\sigma_{\text {Adaptive }}$ is the adaptive standard deviation of the window. The adaptive standard deviation method for each window is given by equation (5).

$$
\sigma_{\text {Adaptive }}=\frac{\sigma_{W}-\sigma_{\min }}{\sigma_{\max }-\sigma_{\min }},
$$

where $\sigma_{\text {Adaptive }}$ is the fixed standard deviation of the window, $\sigma_{W}$ is the standard deviation of the window, $\sigma_{M i n}$ is the minimum standard deviation value and $\sigma_{M a x}$ is the maximum standard deviation value of all windows in the document image. We calculate $\sigma_{\text {Adaptive }}$ to represent the most optimum $\sigma$ value among all windows in an image. This $\sigma_{\text {Adaptive }}$ value can relatively change according to the natural of the image. It gives an idea about nature of the image contrast. Based on our experiments, sometimes the standard deviation values $\sigma$ is insignificant applying on brighter or low contrast images. Therefore in some cases, that drives to inapplicable/ ineffective standard deviation values. For that reason, we adaptive the standard deviation values of each image in the range of $[0,1]$. Based on this $T$ values, the binarization process is defined in Equation (6).

$$
\mathbf{I}(\mathbf{x}, \mathbf{y})=\left\{\begin{array}{lr}
\text { black, } & \mathbf{i}(\mathbf{x}, \mathbf{y})<\mathbf{T}_{\mathbf{W}} \\
\text { white }, & \text { Else }
\end{array}\right.
$$

where $I(\mathrm{x}, \mathrm{y})$ is the image and $i(x, y)$ is the input pixel value of the image. 


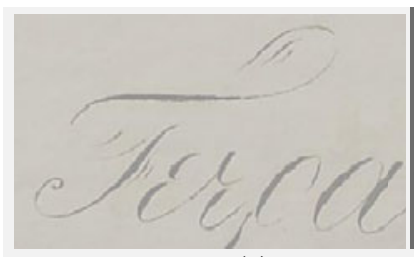

(a)

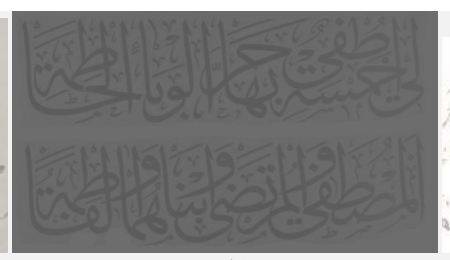

(b)

(c)

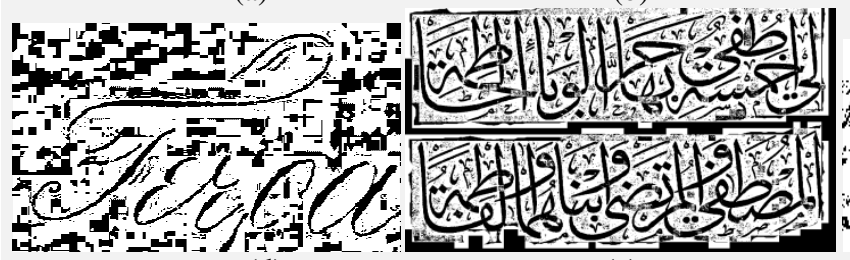

(d) (e)

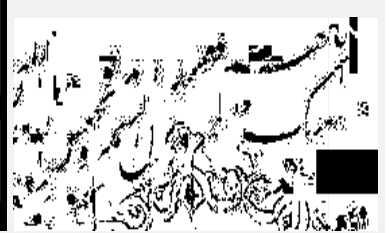

(f)

(g)

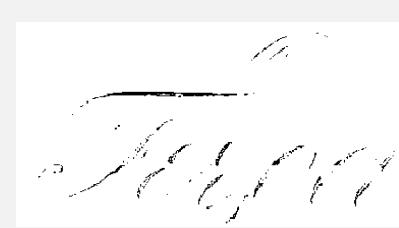

(j)

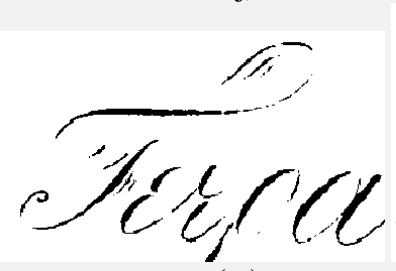

(m) (h)

(k)

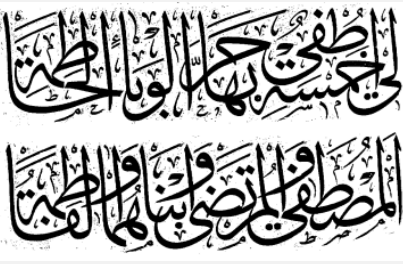

(n)

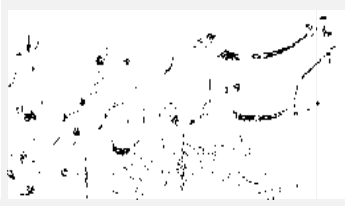

(i)

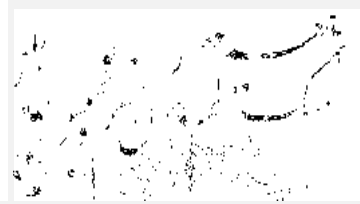

(1)

Fig. 2. (a) is a thin pen stroke (b) and (c) are low contrast image. (d),(e) and (f) are the binarization results of the Niblack's method, (g), (h) and (i) are the binarization results of the Sauvola's method, (j), (k) and (l) are the binarization results of the NICK method (m), (n) and (i) are the results of the proposed method.

\section{The Experiments and Results}

We organize the experiments into two phases; training and testing. In the training phase, we observe relationship between factors and the performance of each method on thin pen stroke text and low contrast images. Then, we identify the most optimum $R, k$ and 
window size for each method. Some of them are a pre-determined factors by the proposed authors. The values identified as: $k=-0.2$ and $25 \times 25$ window size for Niblack method [1], $k=0.2, R=128$ and $15 \times 15$ window size for Sauvola method [8], and $k=-0.2$ and $19 \times 19$ window size for Nick method [5] while $20 \times 20$ window size for the proposed method. Below in Table 1 the parameters setting for each method. Some of visual results are shown according to Niblack's method, Sauvola's method, NICK and proposed method successively in Fig. 2.

Table 1. The factors values of the Niblack, Sauvola, NICK and proposed methods

\begin{tabular}{lllll}
\hline & Niblack [7] & Sauvola [8] & Nick [5] & proposed \\
\hline $\mathbf{k}$ & -0.2 & 0.2 & -0.2 & - \\
$\mathbf{R}$ & - & 128 & - & - \\
Window size & $25 \times 25$ & $15 \times 15$ & $19 \times 19$ & $20 \times 20$ \\
\hline
\end{tabular}

To give a clearer perception of the performance of previous methods, the previous methods have been tested on benchmark dataset and evaluation technique. We test and evaluate existing methods based on benchmark dataset namly Document Image Binarization Contest (DIBCO, 2009), it was prepared for International Conference on Document Analysis and Recognition ICDAR 2009 [9, 10]. This dataset [11] contains 10 document images. These images are color and gray-scale also divided into 5 handwritten and 5 printed document images. These dataset included the general challenges of binarization process. The evaluation technique are based on the $F$-mean measurement, it is available in [12]. As below, the F-mean denotes to the persentage of the binary image accourcy.

$$
\text { F-mean }=2 \times \text { Recall } \times \text { Precision } / \text { Recall }+ \text { Precision, }
$$

where, Recall $=T P / T P+F N$, Precision $=T P / T P+F P$ and, $T P$ is the true-positive value, $F N$ is the false-positive value and $F P$ false-negative values.

Three experiments conducted on DIBCO, (2009) dataset [11]. The first experiment conducted based on selected samples containing thin pen stroke and low contrast problems, the selected samples are: H01 and H05. As shown in Table 2 and Fig. 3, the results of the proposed method is better than other methods. The average of the F-mean are $82.425 \%$ for the proposed method. While $24.883 \%, 33.342 \%$ and $73.6145 \%$ for the Niblack, Sauvola, and NICK methods successively.

Table 2. The F-mean of the Niblack, Sauvola, NICK and proposed methods of the selected samples

\begin{tabular}{llll}
\hline & H01 & H05 & Average \\
\hline proposed & $82.123 \%$ & $82.727 \%$ & $82.425 \%$ \\
Niblack [7] & $32.086 \%$ & $17.68 \%$ & $24.883 \%$ \\
Sauvola [8] & $18.53 \%$ & $48.154 \%$ & $33.342 \%$ \\
NICK [5] & $71.015 \%$ & $76.214 \%$ & $73.6145 \%$ \\
\hline
\end{tabular}




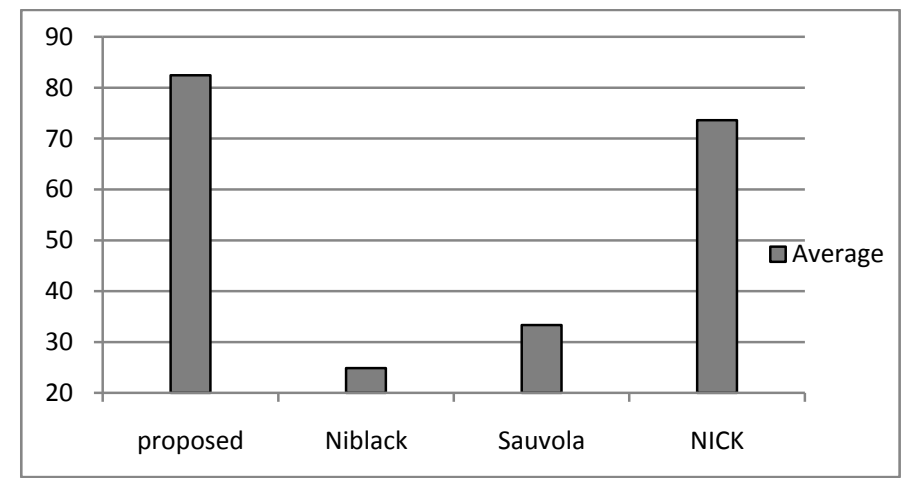

Fig. 3. The F-mean average of the proposed, Niblack, Sauvola and NICK methods

To view the performance of methods on all types of challenges. The next experiment was conducted on dataset images. As exhibited in Table 3 and Fig. 4, the results of the proposed method on both hand written or printed written are higher than the other methods. In the printed written category, the highest F-mean the Niblack's value was achieved by the proposed method about $83.3416 \%$. while the rest achieved about $83.3416 \%, 71.7396 \%$ and $52.363 \%$ to NICK, Sauvola and Niblack methods. Retrieving to previous, we can conclude the average performance for both categories are $84.968 \%, 80.0546 \%, 63.25 \%$ and $38.97 \%$ with respect to the proposed, NICK, Sauvola and Niblack methods subsequently.

Table 3. The F-mean of the Niblack, Sauvola, NICK and proposed methods with autrhers* parameters values

\begin{tabular}{llll}
\hline & $\begin{array}{l}\text { Hand written F- } \\
\text { mean }\end{array}$ & $\begin{array}{l}\text { Printed written F- } \\
\text { mean }\end{array}$ & Average \\
\hline proposed & $82.82 \%$ & $87.12 \%$ & $84.97 \%$ \\
Niblack [7] & $25.57 \%$ & $52.36 \%$ & $38.97 \%$ \\
Sauvola [8] & $54.77 \%$ & $71.74 \%$ & $63.25 \%$ \\
NICK [5] & $76.77 \%$ & $83.342 \%$ & $80.1 \%$ \\
\hline
\end{tabular}

For the results of methods' performance without the influence of windows size. In the following experiment, all methods have applied on the same windows size. The 20 $\times 20$ window size was chosen because it is the more optimum windows size to all methods. As exhibited in Table 4 and Fig. 5, the average of the F-mean results of the proposed method are higher than the other methods. The average of the F-mean is $84.968 \%$ for the proposed method. However, $39.5853 \%, 66.9045 \%$ and $80.0546 \%$ are F-mean results for the Niblack method, Sauvola's method, and NICK method successively. 


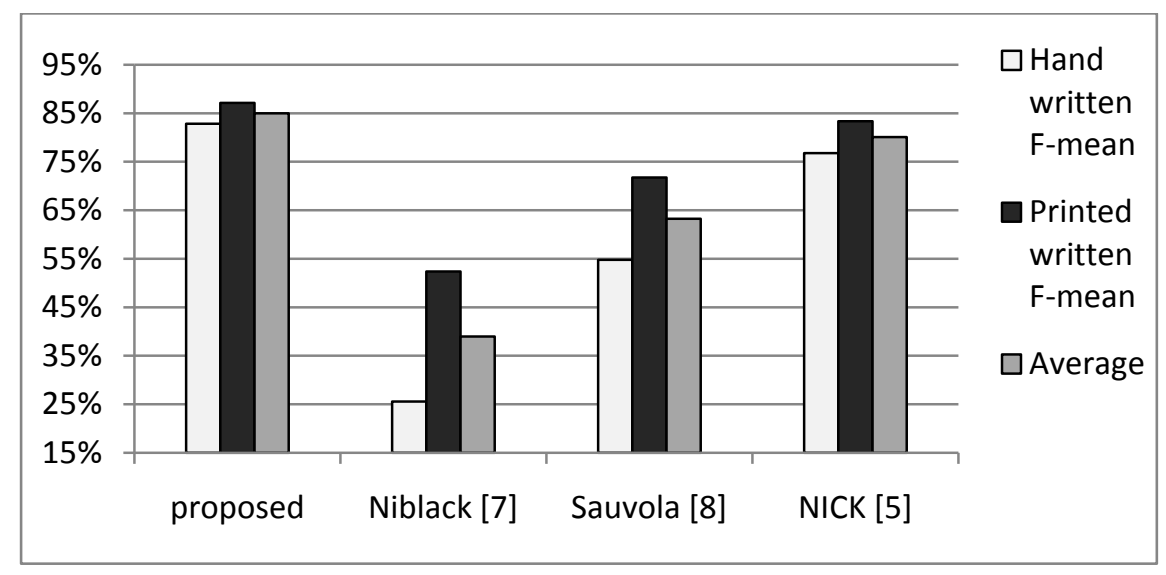

Fig. 4. The F-mean of the proposed, Niblack, Sauvola and NICK methods with autrhers ' parameters values

Also, The proposed method gave the second best performance among each method in each of Recall (the accuracy rate of the text body in the result out image) and Precision (the percentage of binarization noise in the result out image). However, Sauvola's method achieved the best performance in Precision term (97.6\%), but it achieved the lowest Recall performance about 55.2\%. On the other hand, Niblack method achieved the best performance in Recall term about $91.9 \%$, whereas, Niblack method achieved the lowest Precision performance is $27 \%$. However, the proposed method achieved a proportional values in terms of Recall and Precision are $83.3 \%$ and $88.4 \%$ successively. Which makes the proposed method gives the best performance in whole binarization process.

Table 4. The F-mean of the Niblack, Sauvola, NICK and proposed methods with $20 \times 20$ window

\begin{tabular}{rrrr}
\hline & Recall & Precision & F-mean \\
\hline proposed & $83.3 \%$ & $88.4 \%$ & $84.968 \%$ \\
Niblack [7] & $91.9 \%$ & $27 \%$ & $39.585 \%$ \\
Sauvola [8] & $55.2 \%$ & $97.6 \%$ & $66.905 \%$ \\
NICK [5] & $74.5 \%$ & $89.5 \%$ & $80.055 \%$ \\
\hline
\end{tabular}

Based on previous experiments, we have found that the proposed method effective with all types of document images. Apart from successfully solving close contrast image problem, the proposed method is also perform better in thin pen stroke text problems. In addition, the proposed method skipped the problem of identifying the factors manually. 


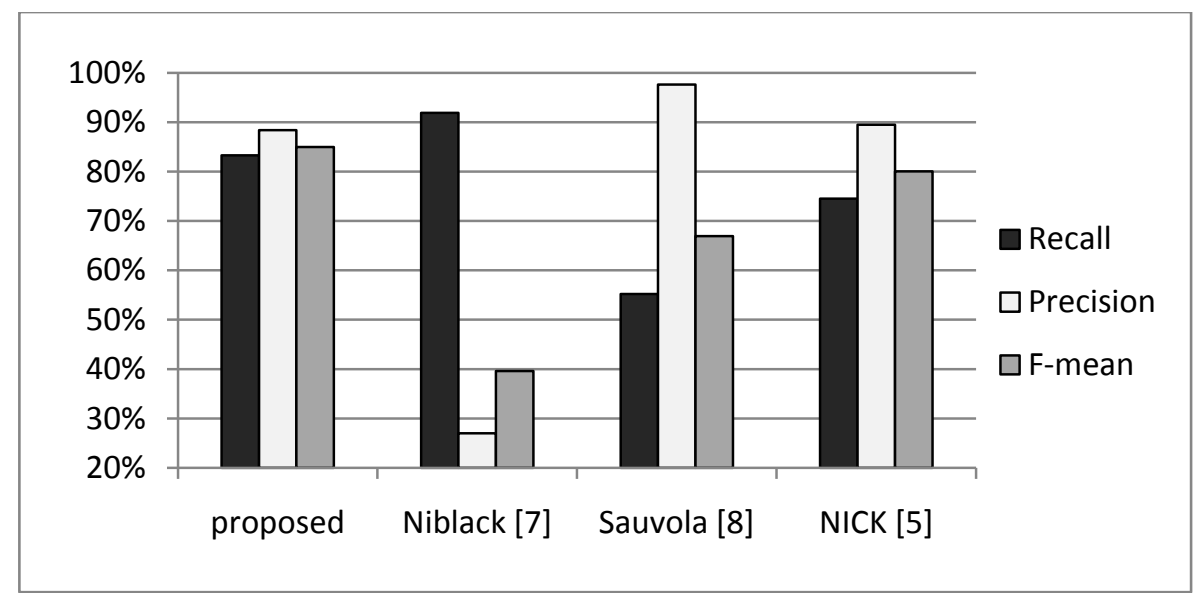

Fig. 5. The F-mean of the proposed, Niblack, Sauvola and NICK methods with $20 \times 20$ window

\section{Conclusion}

The objective of this work is to propose an enhanced binarization method based on local thresholding approach. It presents a new thresholding method that can determine effective threshold values for each windows regions on the document images. The proposed method can deal with all kinds of challenges including the low contrast images and thin pen stroke text. Also, it is skipped the problem of identifying the parameters of the previous methods manually. In the experiments, we have evaluated the proposed method by comparing Niblack's, Sauvola's, and NICK methods. The experiments was conducted on selected documents images and benchmark dataset that dedicated to the problems of the of binarization. In summary, the proposed method has given better performance in comparison to other state of the art methods. Also, it is easy to implement and deals with binarization challenges types.

Acknowledgments. Special thanks are due to Dr. Khurram Khurshid, University Paris Descartes, France, for his assistance and cooperation. This project research was funded by the UKM-TT-03-FRGS0129-2010 grant entitled "Determining adaptive threshold for image segmentation", UKM-TT-03-FRGS0130 entitled "Automatic Background and Subtraction for Image Enhancement" .

\section{References}

1. Kefali, A., Sari, T., Sellami, M.: Evaluation of several binarization techniques for old Arabic documents images. In: The First International Symposium on Modeling and Implementing Complex Systems MISC 2010, Constantine, Algeria, pp. 88-99 (2010)

2. Stathis, P., Kavallieratou, E., Papamarkos, N.: An Evaluation Technique for Binarization Algorithms. Journal of Universal Computer Science 14(18), 3011-3030 (2008) 
3. Gatos, B., Pratikakis, I., Perantonis, S.J.: Adaptive degraded document image binarization. Pattern Recognition 39(3), 317-327 (2006)

4. Otsu, N.: A threshold selection method from gray-level histogram. IEEE Transactions on Systems, Man and Cybernetics 9(1), 62-66 (1979)

5. Khurshid, K., Siddiqi, I., Faure, C., Vincent, N.: Comparison of Niblack inspired Binarization methods for ancient documents. In: 16th International conference on Document Recognition and Retrieval. SPIE, USA (2010)

6. Ntogas, N., Ventzas, D.: A Binarization Algorithm For Historical Manuscripts. In: Proceedings of the 12th WSEAS international conference on Communications, Heraklion, Greece, pp. 41-51 (2008)

7. Niblack, W.: An introduction to digital image processing (1985)

8. Sauvola, J., Seppanen, T., Haapakoski, S., Pietikainen, M.: Adaptive document binarization. In: Fourth International Conference Document Analysis and Recognition (ICDAR), Ulm, Germany (1997)

9. Gatos, B., Ntirogiannis, K., Pratikakis, I.: DIBCO 2009: document image binarization contest. International Journal on Document Analysis and Recognition (2009)

10. Gatos, B., Ntirogiannis, K., Pratikakis, I.: ICDAR 2009 Document Image Binarization Contest. In: $10^{\text {Th }}$ International Conference on Document Analysis and Recognition, Beijing, China (2009)

11. Document Image Binarization Contest (DIBCO 2009), National Center for Scientific Research. Demokritos, Greece (September 2010), http://www.iit.demokritos.gr/ bgat/DIBCO2009/benchmark

12. Document Image Binarization Contest (DIBCO 2009), National Center for Scientific Research. Demokritos, Greece (September 2010),

http://users.iit.demokritos.gr/ bgat/DIBCO2009/

Evaluation.html 